Research Article

\title{
A Form Finding Method for Arch Bridges Using Parametric Level Set Method
}

\author{
Yadong Shen $(\mathbb{D}$, Jianhu Feng $(\mathbb{D}$, Xiaohan Cheng $\mathbb{D}$, Xuntao Wang $\mathbb{D}$, \\ and Changhao Zhang $(1)$
}

\author{
School of Science, Chang'an University, Xi'an, Shaanxi 710064, China \\ Correspondence should be addressed to Jianhu Feng; 2015012001@chd.edu.cn
}

Received 9 July 2018; Accepted 11 October 2018; Published 11 November 2018

Academic Editor: Evangelos J. Sapountzakis

Copyright (C) 2018 Yadong Shen et al. This is an open access article distributed under the Creative Commons Attribution License, which permits unrestricted use, distribution, and reproduction in any medium, provided the original work is properly cited.

\begin{abstract}
Topology optimization has developed rapidly in the past three decades; as a creative and efficient optimization technique, it has been applied in engineering fields of aerospace and mechanical. However, there are a few attempts in bridge form design. In this paper, the parametric level set method is utilized to solve the form finding of arch bridges. The optimization model for minimizing the structural compliance under the volume constraint is built. Three numerical examples of form finding of arch bridges are studied. Results show that the optimal structures which have well-distributed stress and smooth force transmission are almost identical with the actual forms of arch bridges. The optimal forms can be treated as alternatives in the preliminary design stage, and topology optimization has a bright prospect in form finding of arch bridges.
\end{abstract}

\section{Introduction}

In conventional process of engineering design, there is blindness that the initial form of structure is achieved according to the knowledge and experience of engineers. Topology optimization refers to achieving optimal distribution of a material in a given design domain and also obtaining the optimal mechanical performance under specified constraints simultaneously. It can provide a novel and reasonable design in the preliminary design stage. Based on these advantages, topology optimization is widely applied in different fields of engineering, for instance, aerospace, mechanical, electromagnetic, and electrochemical [1-4].

Topology optimization has been developed rapidly in the past three decades. Numerous topology optimization methods including homogenization method $(\mathrm{HM})[5,6]$, density method (DM) [7-9], evolutionary structural optimization (ESO) $[10,11]$, and level set method (LSM) $[12,13]$ have been proposed. Compared with the previous three methods, LSM has a smooth boundary which contains a lot of geometry information, and it is easier to convert the optimal configuration into the CAD model for manufacturing. In addition, LSM handles complex boundary change easily. LSM refers to using of the zero isosurfaces of the level set functions to represent the structural boundaries. In conventional LSM, the evolution of structural boundaries is tracked by solving a Hamilton-Jacobi partial differential equation (H-J PDE). An upwind scheme was employed by Mei et al. [14] to solve the H-J PDE using the discrete solution. In this method, the accuracy and rate of convergence are affected as they are often too steep or too flat near the boundaries during the evolution of structural boundaries. Periodically, reinitializing the signed distance function is needed to alleviate numerical instability. And the optimal result generally depends on the initial topology because the new holes cannot be created in the design domain in the most conventional LSM.

Parametric level set method (PLSM) solves these problems effectively without directly solving the H-J PDE. In this method, the implicit level set function is interpolated by radial basis functions (RBFs), the H-J PDE is converted into a series of ordinary differential equations which are easier to handle, and the propagation of boundaries is driven by updating interpolated coefficients using mathematical programming methods. The PLSM not only inherits the favorable features of the conventional LSM but also avoids the difficulties in solving the HJ-PDE. Wang et al. [15] firstly 
introduced globally supported radial basis functions (GSRBFs) into topology optimization of minimizing the structural compliance under the volume constraints. Luo et al. [16] applied the compactly supported radial basis functions (CSRBFs) [17, 18] into topology optimization of compliant mechanisms and established the optimization model with a nonconvex objective function and specified constraints. The evolution of the boundary was the process of updating the interpolated coefficients using the method of moving asymptotes (MMA) [19]. Luo et al. [20, 21] also proposed an effective method for structural stiffness design which combines the CSRBFs with well-established optimization algorithms, such as MMA and OC.

Up to date, topology optimization has been widely used in fields of aerospace, mechanical, etc. However, it has seen little use in form finding of bridge. Chen et al. [22] obtained the optimal form of arch bridge using BESO and discussed the optimization results with different rise-to-span ratios. Felicetti et al. [23] applied BESO to bridge design in consideration of different requirements, for instance, support types, constraint types, and selections of span. However, this method has the numerical problem of checkerboard. Furthermore, the process of converting the optimized result to the CAD model needs a lot of postprocessing efforts.

In this paper, PLSM which has a lot of geometry information is applied into form finding of arch bridges. The optimization model of minimizing the structural compliance under the volume constraint is proposed. And three examples of arch bridges are studied. The paper is composed as follows. In Section 2, level set representation and parameterization using CSRBF are presented. After that, the topology optimization model is formulated. The sensitivity analysis and the flowchart of optimization are given in Section 3. Section 4 discusses the results of three examples in form finding of arch bridges. Finally, some conclusions are presented in Section 5 .

\section{Parametric Level Set Method}

2.1. Level Set Representation. In the level set method, the structural boundaries are represented by the zero-level set of a higher dimensional level set function, which is usually defined as follows:

$$
\begin{cases}\Phi(x)>0, & \forall x \in \Omega \backslash \Gamma \\ \Phi(x)=0, & \forall x \in \Gamma \\ \Phi(x)<0, & \forall x \in D \backslash(\Omega \cup \Gamma),\end{cases}
$$

where $\Phi(x)$ is the level set function, $D$ is the design domain, $\Omega$ is the positive value of the level set function, and $\Gamma$ denotes the zero-level set.

A pseudotime $t$ is introduced to manage the evolution of structural boundaries; differentiating both the sides of $\Phi(x(t), t)=0$ with respect to $t$, we can obtain that

$$
\frac{\partial \Phi(x, t)}{\partial t}-v_{n}|\nabla \Phi(x, t)|=0,
$$

where $v_{n}$ is the normal velocity of the level set function.

2.2. Parameterization Using CSRBF. The PLSM with compactly supported radial basis function (CSRBF) [20, 21] which has sufficiently smooth and accurate to approximate the level set function is applied into topology optimization of arch bridge. The level set function can be expressed as

$$
\Phi(x, t)=\sum_{i=1}^{N} w_{i} \alpha_{i}(t)
$$

where $N$ is the total number of computational points, $w_{i}$ denotes the CSRBF at the $i$ th point, and $\alpha_{i}$ is the interpolated coefficient at the $i$ th point.

In this work, CSRBF with C2 continuity $[17,18]$ which has strictly positive definiteness and the sparseness of collection matrices is employed to approximate the level set function, and it can be expressed as

$$
w_{i}(x)=\max \left\{0,\left(1-r_{i}(x)\right)\right\}^{4}\left(4 r_{i}(x)+1\right), \quad i=1,2, \ldots, N,
$$

where $r$ is the radius of support, and it is usually defined in a $2 \mathrm{D}$ Euclidean space as

$$
r_{i}(x)=\frac{\left\|x-x_{i}\right\|}{R}, \quad i=1,2, \ldots, N,
$$

where $x_{i}$ is the coordinates of the $i$ th computational point and $R$ is the radius of support domain of the computational point $x$, which is defined as $2.0-4.0$.

By substituting Equation (3) into Equation (2), the HJPDE in Equation (2) can be rewritten as follows:

$$
\sum_{i=1}^{N} w_{i}(x) \dot{\alpha}_{i}(t)-v_{n} \sum_{i=1}^{N}\left|\nabla w_{i}(x)\right| \alpha_{i}(t)=0 .
$$

The normal velocity of the level set function can be calculated by

$$
v_{n}=\frac{\sum_{i=1}^{N} w_{i} \dot{\alpha}_{i}(t)}{\sum_{i=1}^{N}\left|\nabla w_{i}\right| \alpha_{i}(t)},
$$

where $\dot{a}_{i}(t)=d a_{i} / d t$.

Equation (7) shows that $v_{n}$ is obtained by the variation of interpolated coefficients, and the shape change problem is transformed to a parameterization problem.

\section{Topology Optimization for Form Finding of Arch Bridges}

3.1. Optimization Model Using PLSM. In this optimization model, the interpolated coefficients of CSRBFs are adopted as design variables, optimization model of minimizing the structural compliance under the volume constraint is proposed, and it can be mathematically expressed as follows: 


$$
\begin{cases}\text { find } & \alpha_{i}, \quad(i=1,2, \ldots, N) \\ \min . & J=\int_{D} \varepsilon(u) D(\Phi) \varepsilon(u) d \Omega \\ \text { s.t. } & a(u, v, \Phi)=l(v, \Phi) \\ & G(\Phi) \leq f V_{0} \\ & \underline{\alpha} \leq \alpha_{i} \leq \bar{\alpha}\end{cases}
$$

where $J$ denotes the objective function, $u$ is the displacement field, and $v$ is the virtual temperature field. $\varepsilon(u)$ is the strain, $V_{0}$ is the volume of design domain, $G(\Phi)=\int_{D} H(\Phi) d \Omega$ is the volume of structure, $f$ denotes the volume constraint, $\underline{\alpha}$ and $\bar{\alpha}$ are the lower and the upper bounds of the design variables, and $D(\Phi)$ is the elastic stiffness which can be calculated as follows:

$$
D(\Phi)=H(\Phi) E,
$$

where $E$ is the material elastic stiffness and $H(\Phi)$ is the Heaviside function. In order to avoid regenerating the element mesh, we employ a smoothed approximation of $H(\Phi)$, which is defined as

$$
H(\Phi)=\left\{\begin{array}{l}
\chi, \quad \Phi \leq-\Delta, \\
\frac{3(1-\chi)}{4}\left(\frac{\Phi}{\Delta}-\frac{1}{3}\left(\frac{\Phi}{\Delta}\right)^{3}\right)+\frac{1+\chi}{2}, \quad-\Delta \leq \Phi \leq \Delta, \\
1, \quad \Phi \geq \Delta,
\end{array}\right.
$$

$$
\delta(\Phi)=\left\{\begin{array}{l}
\frac{3(1-\chi)}{4 \Delta}\left(1-\left(\frac{\Phi}{\Delta}\right)^{2}\right), \quad|\Phi| \leq \Delta, \\
\gamma, \quad|\Phi| \geq \Delta,
\end{array}\right.
$$

where $\chi$ and $\gamma$ are small positive numbers to avoid singularity of stiffness matrix and $\Delta$ is the width of the numerical approximation.

$a(u, v, \Phi)=l(v, \Phi)$ is the weak form of the state equation, and the energy bilinear form $a(u, v, \Phi)$ and the load linear form $l(v, \Phi)$ can be expressed as follows:

$$
\begin{aligned}
a(u, v, \Phi) & =\int_{D} \varepsilon(u) D(\Phi) \varepsilon(v) d \Omega, \\
l(v, \Phi) & =\int_{D} p v H(\Phi) d \Omega+\int_{D} c v \delta(\Phi)|\nabla \Phi| d \Omega,
\end{aligned}
$$

where $p$ is the body force and $c$ is the boundary traction.

3.2. Sensitivity Analysis. The optimization problem in Equation (8) can be solved by sensitivity-based optimization algorithms. Thus, the derivatives of the objective function and volume constraints with respect to the design variables are required. The Lagrangian method is employed to transform the constrained optimization problem into an unconstrained problem, which can be expressed as

$$
\begin{aligned}
L= & J(u, \Phi)+(a(u, v, \Phi)-l(v, \Phi)) \\
& +\lambda\left(G(\Phi)-f V_{0}\right),
\end{aligned}
$$

where $\lambda$ is the Lagrangian multiplier.
The material derivative is used to obtain the shape derivative of $L$. For more detailed theoretical proofs, the readers can refer to $[20,21]$. The shape derivative of $L$ can be obtained by

$$
\begin{aligned}
\frac{\partial L(u, \Phi)}{\partial t}= & \sum_{i=1}^{N}\left(\int_{D} \beta(u, v, \Phi) w_{i} \frac{\partial \alpha_{i}}{\partial t} d \Omega\right) \\
& +\lambda \sum_{i=1}^{N}\left(\int_{D} \delta(\Phi) \frac{\partial \alpha_{i}}{\partial t} d \Omega\right),
\end{aligned}
$$

and

$$
\begin{aligned}
\beta(u, v, \Phi)= & f(u, u)-\varepsilon(u) C \varepsilon(v) \\
& +[p v+\nabla(c v) \bullet \mathrm{n}+\kappa(c v)],
\end{aligned}
$$

where $\kappa=\operatorname{divn}=\nabla \bullet(-\nabla \Phi /|\nabla \Phi|)$ is the mean curvature.

The derivative of Lagrangian $L$ with respect to $t$ can also be obtained by the chain rule as follows:

$$
\frac{\partial L(u, \Phi)}{\partial t}=\left(\sum_{i=1}^{N} \frac{\partial J(u, \Phi)}{\partial \alpha_{i}}+\lambda \sum_{i=1}^{N} \frac{\partial G(\Phi)}{\partial \alpha_{i}}\right) \frac{\partial \alpha_{i}}{\partial t} .
$$

By comparing the corresponding terms in Equations (14) and (16), the derivatives of the objective function and the volume constraint with respect to the interpolated coefficients can be obtained by

$$
\begin{aligned}
\frac{\partial J(u, \Phi)}{\partial \alpha_{i}} & =\int_{D} \beta(u, v, \Phi) w_{i} d \Omega, \\
\frac{\partial G(\Phi)}{\partial \alpha_{i}} & =\int_{D} \delta(\Phi) d \Omega .
\end{aligned}
$$

When Equations (17) and (18) are obtained, the optimization problem in Equation (8) can be solved by sensitivity-based optimization algorithms.

3.3. Optimization Procedure. In optimization problem, with the sensitivities of objective function and volume constraint with respect to interpolated coefficients obtained, the sensitivity-based optimization algorithms such as optimality criteria and mathematical programming can be applied straightforwardly. Then, the shape and topology optimization problem converts into the parameter optimization problem. In this work, the method of moving asymptotes (MMA) which is a widely used mathematical programming algorithm is employed to solve the optimization problem. The process of optimization is shown in Figure 1, and the corresponding introduction is presented as follows:

Step 1: initialize the design domain of arch bridge. Define the load, boundary conditions, and set the volume constraint. 




Figure 1: Flowchart of form finding of arch bridge.

Step 2: FE analysis and calculate the real and virtual displacement fields.

Step 3: calculate the derivatives of objective function and the volume constraint with respect to the interpolated coefficients in Equations (17) and (18).

Step 4: calculate the values of objective function and the volume constraint. Update the interpolated coefficients with MMA optimization algorithm, and then, the update level set function.

Step 5: check whether the convergence condition is shown in Equation (19). If the terminal condition is met, stop, and the optimal structure is found. Otherwise, go back to Step 2. The terminal condition is defined as

$$
\left|\frac{J^{(s)}-J^{(s-1)}}{J^{(s)}}\right| \leq \varepsilon
$$

where $J^{(s)}$ denotes the value of the objective function at sth iteration, and $\varepsilon$ is a small positive value.

\section{Numerical Examples}

In this section, form finding of three kinds of arch bridges is presented to illustrate the effectiveness of the proposed method. The conventional level set method (CLSM), BESO, and SIMP are applied for comparison. In these examples, the computational points in PLSM are corresponding to the finite element nodes and four-node plane stress elements are used for finite element analysis (FEA). The parameters in
Equation (8) are $\bar{\alpha}^{k}=6 \times \max \left(\alpha_{i}^{k}\right)^{0}$ and $\underline{\alpha}^{k}=6 \times \min \left(\alpha_{i}^{k}\right)^{0}$, where $\left(\alpha_{i}^{k}\right)^{0}$ denotes the initial values of interpolation coefficients, and the support radius for computational points is set to be $R=2.5 . \Delta=1, \chi=0.001$, and $\gamma=0.0005$ are the parameters in Equations (10) and (11). The parameters in BESO are $E R=2 \%, A R \max =50 \%$, and $\mathrm{rmin}=2.5$, while in SIMP, $r \min =2.5$. The Lenovo personal computer equipped with 1 Intel $^{@}$ Core $^{T M}$ i5-3230M processor with a clock speed of $2.6 \mathrm{GHz}$ and $4.00 \mathrm{~GB}$ RAM is employed to calculate central processing unit (CPU) times.

Example 1. In this deck arch bridge design example, the admissible design domain, boundary conditions, and load conditions are shown in Figure 2. The design domain is modeled into $180 \times 60$ elements, a uniform load $F=0.01$ is applied in the top of the design domain, and the prescribed volume fraction is $f=0.3$.

The optimal layouts obtained by PLSM, CLSM, BESO, and SIMP are shown in Figures 3(a)-3(d), respectively, and it can be seen that the optimal layouts of different methods are almost identical and they are similar to the actual layout of deck arch bridge, which shows the effectiveness of PLSM. However, they also have some differences. The optimal layouts of PLSM and CLSM in Figures 3(a) and 3(b) have smooth boundaries, which are easier to transform into CAD model for bridge design. Figure 3(c) shows the optimal layout of BESO with the zigzag boundary. It is hard for follow-up design. Figure 3(d) shows the optimal layout of SIMP. There are a lot of gray elements, and the bars have split forks in the junction point of the bars and the bridge 




Figure 2: Design space of the deck arch bridge.

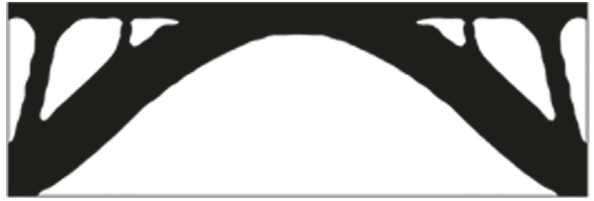

(a)

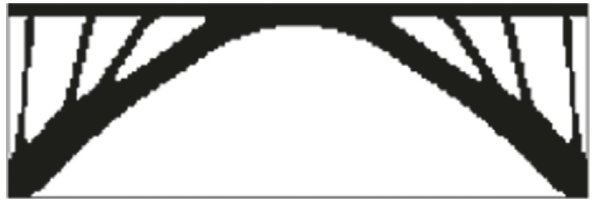

(c)

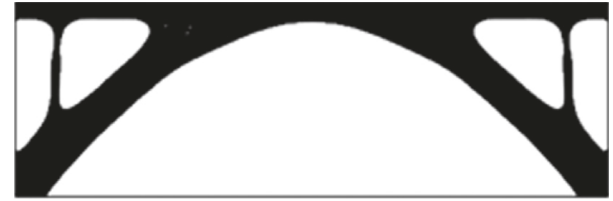

(b)



(d)

FIgURE 3: The optimal layout of deck arch bridge by different methods: (a) PLSM, (b) CLSM, (c) BESO, and (d) SIMP.

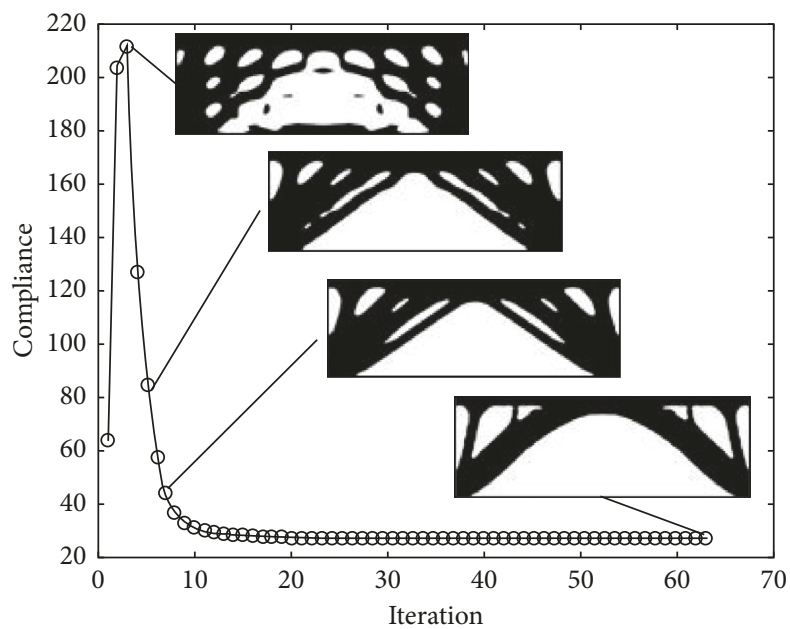

FIGURE 4: Iteration history of structural compliance for form finding of deck arch bridge.

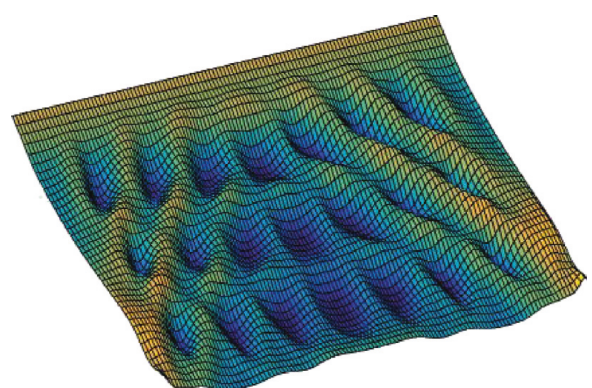

(a)

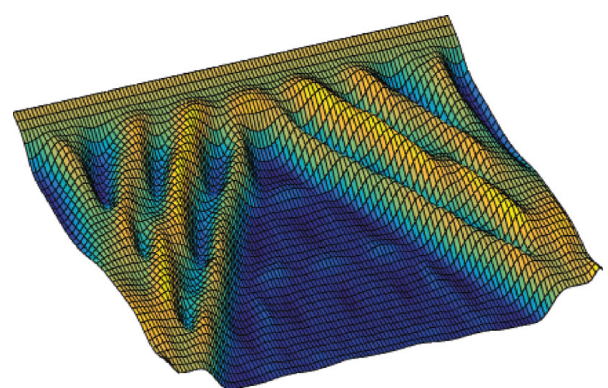

(b)

Figure 5: Continued. 


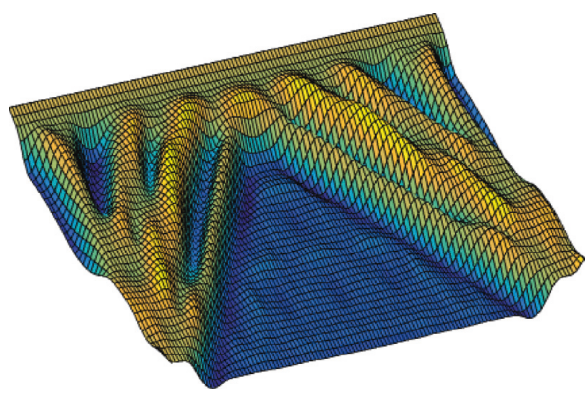

(c)

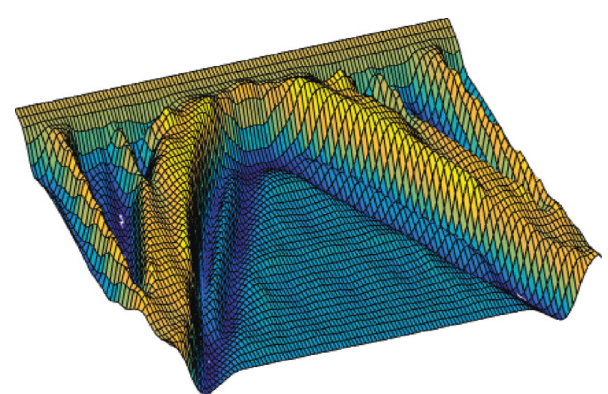

(d)

Figure 5: Level set surfaces at different iterations.

deck, which shows the optimal transmission path of force. In addition, the relatively smooth boundary can be produced by BESO and SIMP when the design space is modeled into a large number of finite elements. In this way, the computational efficiency is greatly reduced.

Figure 4 gives the iteration history of the objective function and several intermediate results. The value of the objective function is 25.2 after 63 iterations. Corresponding to the PLSM topological designs in Figure 4, the level set surfaces at different iterations are displayed in Figure 5. Table 1 provides the results of different methods in form finding of deck arch bridge for comparison. It can be found that the compliance of PLSM is almost the same with that of other methods. And BESO produces the lowest compliance of 24.6 as it utilizes discrete variables. The PLSM costs the shortest computation time of $55.3 \mathrm{~s}$ which shows the high computational efficiency.

Example 2. In this half-through arch bridge design example, the admissible design domain, boundary conditions, and load conditions are shown in Figure 6. The design domain is modeled into $180 \times 120$ elements, a uniform load $F=0.01$ is applied in the height of $h=30$, and the prescribed volume fraction is $f=0.2$.

Figure 7 shows the optimal layouts obtained by different optimization methods. It can be seen that the optimal layouts of different methods are similar to the actual layout of half-through arch bridge. The optimal configuration in PLSM is almost identical with the optimal configuration in CLSM as they have the same number of bars, which shows the validity of PLSM. Both of them have smooth boundaries. The optimal configurations of BESO and SIMP have more bars than that of PLSM and CLSM. The optimal configuration obtained by BESO in Figure 7(c) has inclined supports under the deck and also has a zigzag boundary. The configuration of SIMP in Figure 7(d) has a lot of gray elements.

The iteration history of the objective function and several intermediate results are shown in Figure 8. The objective function is convergent to 19.9 after 37 iterations. For comparison, the results of different methods in form finding of deck arch bridge are presented in Table 2. It can be found that the compliance of PLSM is less than that of CLSM and SIMP, and BESO produces the lowest compliance of 18.6. The PLSM costs the shortest computation
TABLE 1: Comparison of results of different methods in form finding of deck arch bridge.

\begin{tabular}{lcccc}
\hline & PLSM & CLSM & BESO & SIMP \\
\hline Compliance & 25.2 & 25.7 & 24.6 & 25.9 \\
Computation time(s) & 85.3 & 284.5 & 253.1 & 193.2 \\
\hline
\end{tabular}

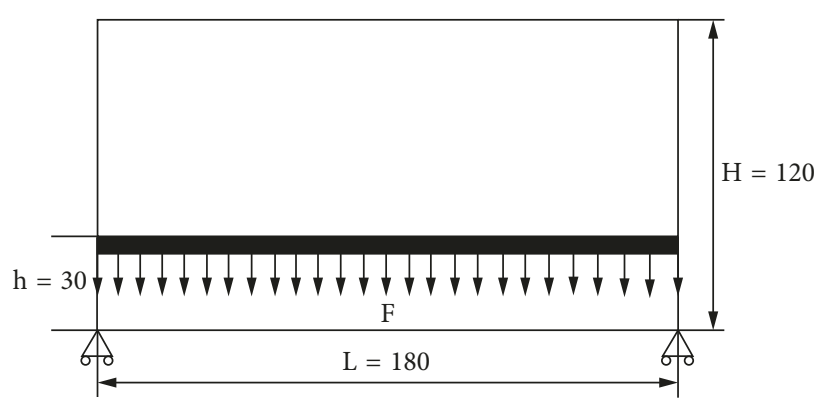

Figure 6: Design space of the half-through arch bridge.

time of $100.9 \mathrm{~s}$ which is much less than other methods in calculation.

Example 3. In this through arch bridge design example, the admissible design domain, boundary conditions, and load conditions are shown in Figure 9. The design domain is modeled into $180 \times 120$ elements, a uniform load $F=0.01$ is applied in the bottom of the design domain, and the prescribed volume fraction is $f=0.2$.

The optimal results of various topology optimization methods are illustrated in Figures 10(a)-10(d), respectively. It can be seen that the optimal layouts of different methods are similar to the actual layout of through arch bridge. The optimal configurations in PLSM and CLSM have smooth boundaries, and the optimal configuration in PLSM is almost identical with the optimal configuration in CLSM. So, the effectiveness of the PLSM can be verified. The optimal configurations of BESO and SIMP have more bars than that of PLSM and CLSM. The configuration of BESO in Figure 10(c) has a zigzag boundary. The configuration of SIMP in Figure $10(\mathrm{c})$ has a lot of gray elements. And, it should be noticed that the bars have split forks in the junction point of the bars and the bridge deck. 


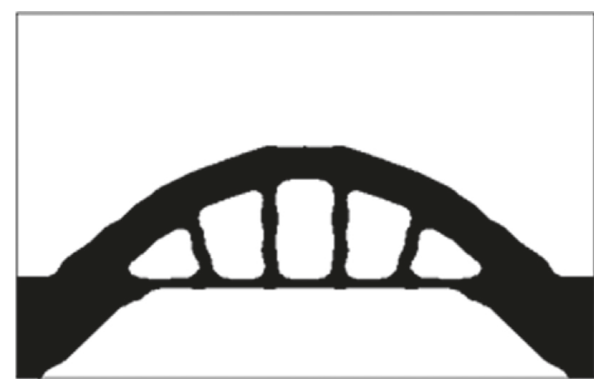

(a)



(c)



(b)

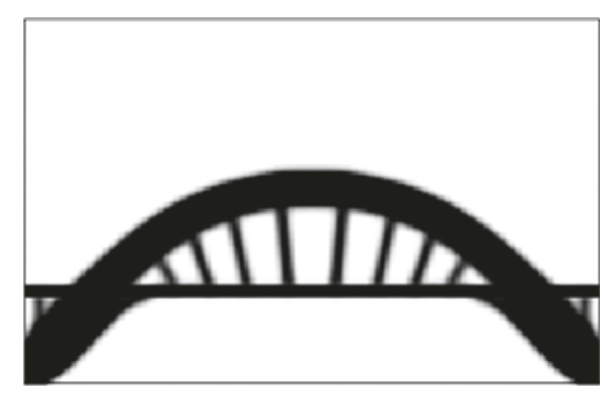

(d)

FIgUre 7: The optimal layout of half-through arch bridge by different methods: (a) PLSM, (b) CLSM, (c) BESO, and (d) SIMP.



FIGURE 8: Iteration history of structural compliance for form finding of half-through arch bridge.

TABLE 2: Comparison of results of different methods in form finding of half-through arch bridge.

\begin{tabular}{lcccc}
\hline & PLSM & CLSM & BESO & SIMP \\
\hline Compliance & 19.9 & 20.7 & 18.6 & 20.1 \\
Computation time(s) & 100.9 & 451.7 & 603.6 & 306.5 \\
\hline
\end{tabular}

Figure 11 gives the iteration history of the objective function and several intermediate results. The value of objective function is 24.3 after 80 iterations. Table 3 summarizes the structural compliances and computation time of different methods in form finding of through arch bridge. It can be found that the compliance of PLSM is almost the same with that of other methods, and BESO produces the lowest compliance of 24.6. The PLSM costs the shortest computation time of 186.2 s. So, the accuracy and efficiency of PLSM are proved.

\section{Conclusions}

In this study, a form finding method for arch bridge is developed using the PLSM-based topology optimization, the solution procedure of the topology optimization method is described, and three numerical examples are employed to illustrate the feasibility and validity of PLSM in form finding of arch bridges. Numerical examples indicate that the configuration obtained by PLSM has smooth boundary. It is easier to convert the configuration to CAD model for manufacturing. In addition, the computational efficiency has been greatly improved. 


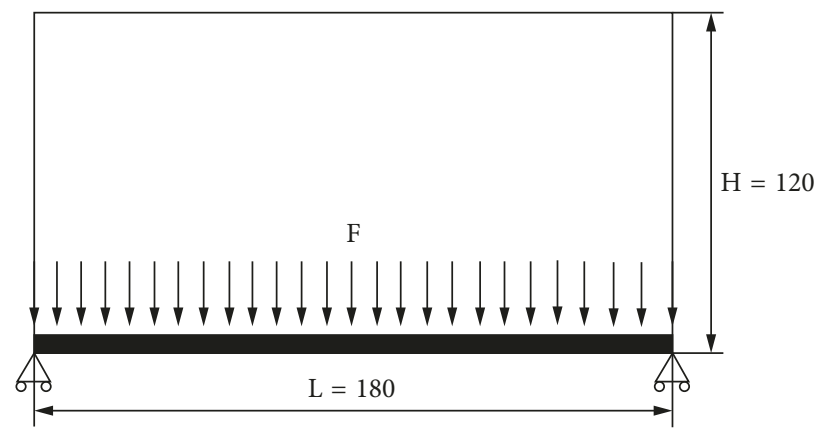

Figure 9: Design space of the through arch bridge.

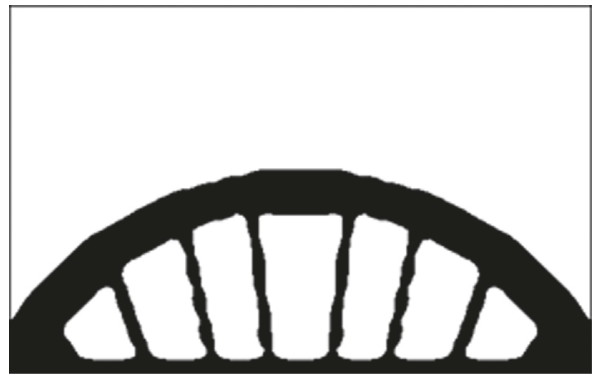

(a)

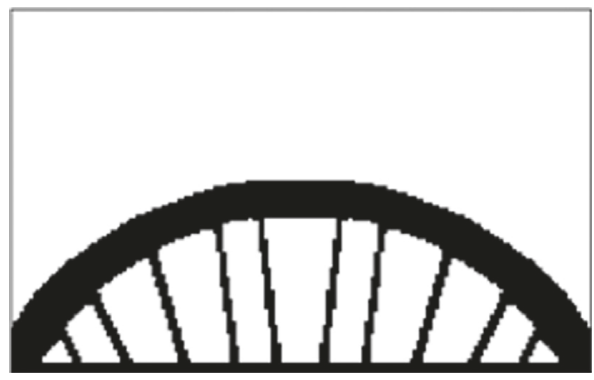

(c)

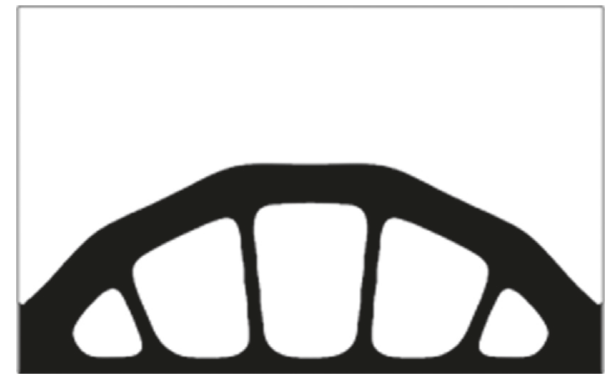

(b)

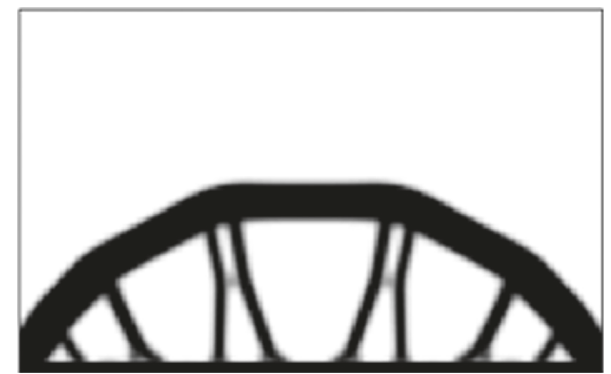

(d)

Figure 10: The optimal layout of through arch bridge by different methods: (a) PLSM, (b) CLSM, (c) BESO, and (d) SIMP.



FIGURE 11: Iteration history of structural compliance for form finding of through arch bridge.
TABLE 3: Comparison of results of different methods in form finding of through arch bridge.

\begin{tabular}{lcccc}
\hline & PLSM & CLSM & BESO & SIMP \\
\hline Compliance & 24.3 & 23.2 & 22.4 & 25.2 \\
Computation time(s) & 186.2 & 432.7 & 503.7 & 389.9 \\
\hline
\end{tabular}

This method also can be extended to form finding of arch bridges with different rise-to-span, main tower of cable stayed bridge, and main cable of suspension bridge. Due to the effectiveness in form finding of arch bridges, it is expected that this method will be applied in bridge design in the future.

\section{Data Availability}

The data used to support the findings of this study are available from the corresponding author upon request. 


\section{Conflicts of Interest}

The authors declare that they have no conflicts of interest.

\section{Acknowledgments}

This work was supported by the National Natural Science Foundation of China (11601037), Shanxi Province Natural Science Fund (2018JQ1027), and the Research Funds for the Central Universities of China (310812171002).

\section{References}

[1] M. P. Bendsøe and O. Sigmund, Topology Optimization: Theory, Methods, and Applications, Springer, Berlin, Germany, 2003.

[2] W. H. Zhang, Z. D. Zhang, J. H. Zhu et al., "Structural topology optimization: extensibility and attainability," Science China Technological Sciences, vol. 57, no. 7, pp. 1310-1321, 2014.

[3] J. H. Zhu, W. H. Zhang, and L. Xia, "Topology optimization in aircraft and aerospace structures design," Archives of Computational Methods in Engineering, vol. 23, no. 4, pp. 595-622, 2016.

[4] P. D. Dunning, B. K. Stanford, and H. A. Kim, "Coupled aerostructural topology optimization using a level set method for 3D aircraft wings," Structural and Multidisciplinary Optimization, vol. 51, no. 5, pp. 1113-1132, 2015.

[5] M. P. Bendsøe and N. Kikuchi, "Generating optimal topologies in structural design using a homogenization method," Computer Methods in Applied Mechanics and Engineering, vol. 71, no. 2, pp. 197-224, 1988.

[6] G. Allaire, F. Jouve, and H. Maillot, “Topology optimization for minimum stress design with the homogenization method," Structural and Multidisciplinary Optimization, vol. 28, no. 2-3, pp. 87-98, 2004.

[7] M. P. Bendsøe, "Optimal shape design as a material distribution problem," Structural Optimization, vol. 1, no. 4, pp. 193-202, 1989.

[8] M. Zhou and G. Rozvany, "The COC algorithm, part II: topological, geometry and generalized shape optimization," Computer Methods in Applied Mechanics and Engineering, vol. 89, no. 1-3, pp. 309-336, 1991.

[9] M. P. Bendsøe and O. Sigmund, "Material interpolation schemes in topology optimization," Archive of Applied Mechanics, vol. 69, no. 9-10, pp. 635-654, 1999.

[10] Y. M. Xie and G. P. Steven, "A simple evolutionary procedure for structural optimization," Computers and Structures, vol. 49, no. 5, pp. 885-896, 1993.

[11] X. Huang and Y. M. Xie, "A further review of ESO type methods for topology optimization," Structural and Multidisciplinary Optimization, vol. 41, no. 5, pp. 671-683, 2010.

[12] M. Y. Wang, X. Wang, and D. Guo, "A level set method for structural topology optimization," Computer Methods in Applied Mechanics and Engineering, vol. 192, no. 1-2, pp. 227-246, 2003.

[13] G. Allaire, F. Jouve, and A. M. Toader, "Structural optimization using sensitivity analysis and a level-set method," Journal of Computational Physics, vol. 194, no. 1, pp. 363-393, 2004.

[14] Y. Mei and X. Wang, "A level set method for structural topology optimization and its applications," Computer Methods in Applied Mechanics and Engineering, vol. 35, no. 7, pp. 415-441, 2004.
[15] S. Wang and M. Y. Wang, "Radial basis functions and level set method for structural topology optimization," International Journal for Numerical Methods in Engineering, vol. 65, no. 12, pp. 2060-2090, 2006.

[16] Z. Luo, L. Tong, M. Y. Wang et al., "Shape and topology optimization of compliant mechanisms using a parameterization level set method," Journal of Computational Physics, vol. 227, no. 1, pp. 680-705, 2007.

[17] H. Wendland, "Piecewise polynomial, positive definite and compactly supported radial functions of minimal degree," Advances in Computational Mathematics, vol. 4, no. 1, pp. 389-396, 1995.

[18] H. Wendland, "Computational aspects of radial basis function approximation," Studies in Computational Mathematics, vol. 12, pp. 231-256, 2006.

[19] K. Svanberg, "The method of moving asymptotes-a new method for structural optimization," International Journal for Numerical Methods in Engineering, vol. 24, no. 2, pp. 359-373, 1987.

[20] Z. Luo, M. Y. Wang, S. Wang et al., "A level set-based parameterization method for structural shape and topology optimization," International Journal for Numerical Methods in Engineering, vol. 76, no. 1, pp. 1-26, 2008.

[21] Z. Luo, L. Tong, and Z. Kang, "A level set method for structural shape and topology optimization using radial basis functions," Computers and Structures, vol. 87, no. 7-8, pp. 425-434, 2009.

[22] A. Chen and C. Chang, "Evolutionary structural optimization in form finding of bridges," Journal of Tongji university (Natural Science), vol. 40, pp. 8-13, 2012.

[23] P. Felicetti, T. Black, X. Huang et al., "Application of topological optimization technology to bridge design," Structural Engineering International, vol. 24, no. 2, pp. 185-191, 2014. 


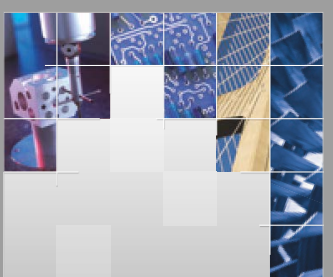

\section{Enfincering}


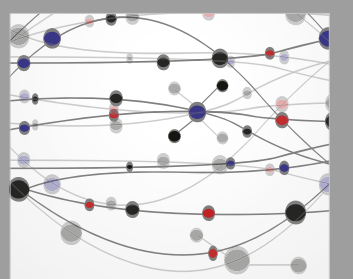

\section{Rotating \\ Machinery}

The Scientific World Journal

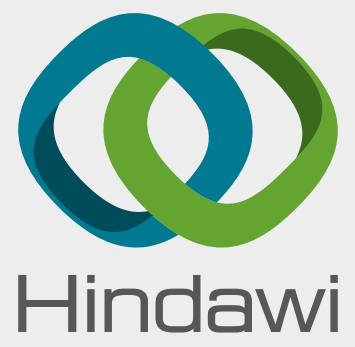

Submit your manuscripts at

www.hindawi.com
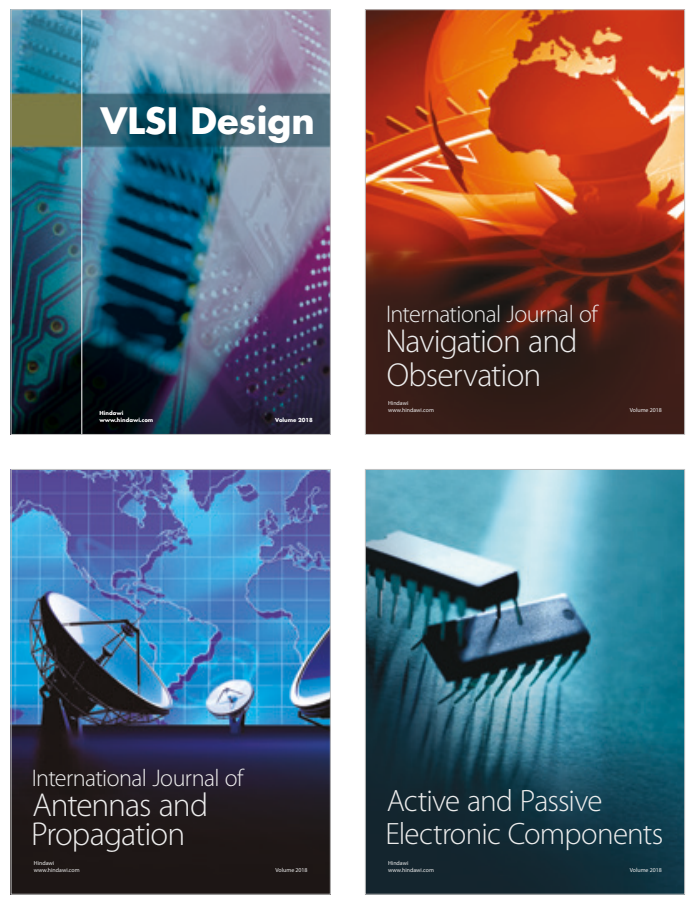
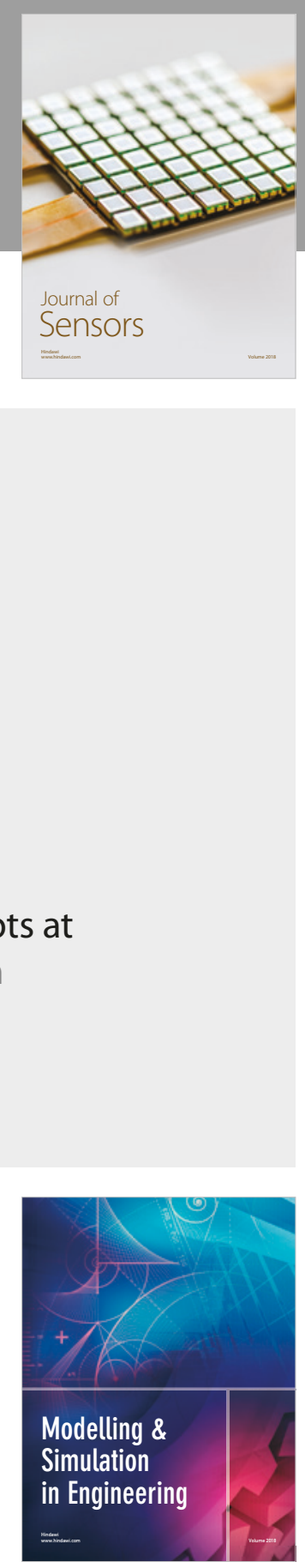

\section{Advances \\ Multimedia}
